\title{
Word sense disambiguation for event trigger word detection in biomedicine
}

\author{
David Martinez ${ }^{1,2^{*}}$, Timothy Baldwin ${ }^{1,2}$ \\ From Fourth International Workshop on Data and Text Mining in Biomedical Informatics (DTMBio) 2010 \\ Toronto, Canada. 26 October 2010
}

\begin{abstract}
This paper describes a method for detecting event trigger words in biomedical text based on a word sense disambiguation (WSD) approach. We first investigate the applicability of existing WSD techniques to trigger word disambiguation in the BioNLP 2009 shared task data, and find that we are able to outperform a traditional CRFbased approach for certain word types. On the basis of this finding, we combine the WSD approach with the CRF, and obtain significant improvements over the standalone CRF, gaining particularly in recall.
\end{abstract}

\section{Introduction}

In recent years, the biomedical text processing field has created many annotated resources to further the development of automatic text analysis methods through standardised evaluation. Following the example of TREC [1] and other efforts, the goal is to develop datasets over which different approaches can be tested and compared. There is considerable diversity in the types of resources that have been produced, from TREC-style document relevance scores to the semantic annotation of all terms in a set of documents (for entities and events of interest).

Different resources annotate data at varying levels of abstraction. In some cases, the target concepts are named entity mentions, such as names of proteins or medications, on which traditional named entity recognition (NER) techniques perform well [2]. In other cases, however, the annotation is over biomedical processes such as events, and the task has been found much harder to tackle [3]. The main problem is that a single class is used to annotate a wide range of linguistic realisations, and NER approaches that rely on recurrences of tokens with predictable labelling are often tripped up. For instance, consider the following examples from the BioNLP 2009 event extraction task [4], where the tags

\footnotetext{
* Correspondence: david.martinez@nicta.com.au

'NICTA VRL, The University of Melbourne, VIC 3010, Australia

Full list of author information is available at the end of the article
}

are shown as sub-indices to the open brackets of the target words:

(1) The [trans changes] in the mRNA levels of these protooncogenes...

(2) The human platelet-activating factor receptor (PAFR) gene is [TRANs transcribed] by...

We can see that two instances have been tagged with the event TRANS (short for TRANSCRIPTION), which refers to the process of creating an equivalent RNA copy of a sequence of DNA. This event is associated with different surface forms in the text: the noun changes in (1) and to the verb transcribed in (2). These types of wide-ranging lexical variations are common across all event types in the BioNLP task, and make both the annotation and the task challenging, with the best system in the shared task obtaining just above 50\% F-score in the basic event structure recognition task (task 1: [4]).

When studying the BioNLP 2009 dataset, we observed that there is a set of high-frequency word types that tend to occur over many event types. This suggests the possibility of building separate models for each of these word types, in a fashion applied in word sense disambiguation (WSD). This technique has not been tested by previous trigger-word detection systems, where the approach is to build event-centred systems, or rely on hand-made dictionaries. The appeal of WSD is that it has been studied widely, and has been shown to perform well under certain conditions: shared tasks like SemEval 
[5] have illustrated that WSD systems can perform above $70 \%$ accuracy for fine-grained sense inventories [6]; and the performance over NLM-WSD, a collection tailored to the biomedical domain, is close to $90 \%$ accuracy [7]. Should we be able to replicate these levels of accuracy over the BioNLP 2009 data, it has the potential to boost overall trigger word detection performance.

Another motivation for combining biomedical datasets and WSD is that the WSD community is constantly looking for new semantically-annotated data to test the portability of their systems over. There are only a few examples of domain-specific corpora annotated with sense information, and they are costly to produce [8]. For instance the NLM-WSD collection [9] was constructed using 11 annotators, and has been used extensively for biomedical WSD experiments. An alternative could be to adapt the semantic annotation of BioNLP and other related biomedical datasets (e.g. BioCreative [10]) in order to generate more testbeds.

To summarise, our main hypothesis in this paper is that biomedical term-annotation tasks can benefit from WSD methods, which we will empirically test over the BioNLP event extraction dataset, where the correct identification of "trigger words" is a crucial component of the overall problem. We will adapt this dataset into a WSD-style collection, and evaluate the performance over a sample of word types that have particular properties, such as having enough training examples and a class distribution that is not overly skewed. We will analyse the raw performance scores to see if we can achieve similar performance to those achieved over other WSD problems, and we will also study the dataset itself, by measuring the strength of the "one sense per collocation" heuristic in relation to other WSD datasets.

The primary findings of this paper are: (a) WSD can indeed outperform sequential tagging techniques over high-frequency terms with relatively low skew; and (b) the overall performance of sequential tagging methods is boosted when we selectively include predictions from our WSD model.

This article is organised as follows. We describe the background of our research in Section. We then introduce our experimental setting in Section, and perform an analysis of feature types in Section. After this study, our main experimental results are presented in Section . We discuss further our experiments and analyse the errors in Section, and finally present our conclusions and future directions in Section .

\section{Background}

In this paper, we focus exclusively on the BioNLP 2009 shared task dataset, where events are defined relative to trigger words of different types, and the goal is to both identify the trigger words and infer the role they play in a given event. There are three separate subtasks in this challenge, and in this paper we focus on Task 1: Core event extraction. Trigger words are linked to a total of 9 events relating to protein biology, which we list in Table 1. The annotation of protein occurrences in the text is given to participants in advance, and these are used as arguments for event triggers.

In the original BioNLP 2009 shared task, most systems relied on at least two separate modules: trigger detection and event construction. Trigger detection involves the identification of event triggers and their type, while event construction associates event triggers with their arguments. For our experiments, we will focus on the trigger detection task, in order to simplify the analysis and comparison of different methods. The systems present in the BioNLP shared task addressed the trigger detection subtask by relying on hand-made dictionaries, sequential classifiers, or class-specific models; not word type models as we do in this paper. The top-ranked system in the 2009 BioNLP shared task was developed by a team from the University of Turku [11]. Their pipeline consists of three main steps: trigger detection, argument assignment, and semantic post-processing. For trigger detection they treat each token as a separate classification problem, and train SVMs for each event type. They rely on a rich set of features, including a dictionary built from the training data, and syntactic dependencies. Their overall task-1 system achieved an F-score of $52 \%$ (with $47 \%$ recall and $58 \%$ precision) by relying on separate SVM classifiers for trigger detection and argument assignment. However, the performance over the trigger detection step in isolation was not reported.

The second-ranked system for the task was built by a team from the University of Jena [12]. Their main architecture also had an independent module for trigger detection, which relied heavily on hand-curated dictionaries, built from the GENIA event corpus [3] and other sources. Their work required manual effort to pre-identify the predictive power of candidate trigger words for each event type, and they relied on this information to build dependency graphs that were refined in subsequent steps. The recall of their system was similar to

\section{Table 1 List of target events for BioNLP 2009}

Gene expression
Transcription
Protein catabolism
Localization
Binding
Phosphorylation
Regulation
Positive regulation
Negative regulation


the top ranked system, but the precision was much lower. Again, their performance for trigger word detection is not known.

We turn to the WSD literature to see if these techniques can contribute to the trigger detection task. There is a large literature on WSD; see [13] and [14] for recent overviews. The most successful approaches are supervised systems that build a separate model for each word type and POS, learning only from contexts that include it. The motivation behind this approach is the "one sense per collocation" heuristic [15], which observes that the meaning of a given word in a particular collocation tends to be invariant across all token occurrences.

One method that has been shown to perform consistently well over open-domain WSD and biomedical WSD is the Vector Space Model (VSM). It achieved high performance over the biomedical NLM-WSD collection (close to 90\% accuracy) [7], and it has also been applied to the Senseval-3 English Lexical Sample dataset [16], where it ranked among the top systems with an accuracy of $72 \%$ [17]. This classifier can accommodate a wide range of features, from local dependencies to $\mathrm{MeSH}$ terms (cf. Section).

\section{Experimental setting}

We designed an experiment to integrate a WSD module into an event-extraction system based on the BioNLP 2009 shared task data. We first describe the dataset used in this experiment, then the different systems tested.

\section{Datasets}

In order to build a WSD collection, we first identified the candidate target words in the BioNLP data that are most likely to benefit from WSD, in terms of both WSD having a good chance of performing well over them, and there being enough token instances that, when fed back into a larger system, the predictions can potentially have a significant impact. Each word occurrence in the data will have one of the 9 trigger-word event classes or the non-event class (a total of 10 classes). Candidate word types for WSD are those which have high frequency and occur with different event classes. We rely on the GENIA tagger [18] for tokenisation and POS-tagging, and we group word occurrences by lemma and POS.

For this experiment we chose the words in the BioNLP 2009 training data that had a token bias lower than $90 \%$ for the majority class (i.e. there are more than $10 \%$ of token instances which occur with a class other than the majority class), and at least 50 training instances. We separate the occurrences of words according to their POS, e.g. we would separate out the noun and verb instances of the word change, and build dedicated WSD classifiers for each. The 90\% threshold filters out word types that have a strong prior for a single class, where there is little margin for improvement. Keeping these high-skew words would boost the performance, but we opted to remove them to focus on the higher-entropy, more interesting cases. The word selection process leaves us with 63 word types, which correspond to 14,903 train instances and 2,910 test instances. Note that we use the development data provided by the task organisers as the test set, since the final test data has not been released. Out of these test instances, 848 belong to annotated events, covering $65 \%$ of the 1,300 trigger event annotations in the test set. If we include the word types with high skew (i.e. $90 \%$ or more), we cover 990 trigger-annotated instances $(76 \%$ of the test events), leaving only $24 \%$ of triggers to be identified with other techniques. The complete list of target words is given in Table 2. We also rely on existing WSD datasets in order to analyse if our new collection has significant differences regarding the class-entropy of the features. For this, we studied two WSD corpora where our WSD method has been shown to perform well: the biomedical NLM-WSD collection, and the open-domain Senseval-3 English Lexical Sample collection. The former consists of 50 word types with 100 annotated token instances each; the latter contains 57 word types, with an average of 132 annotated token instances each. Our own BioNLP dataset has an average of 282 token instances per word type.

\section{Classifiers and features}

Our main WSD classifier is based on the Vector Space Model (VSM), in the form of a nearest-prototype classifier. Each occurrence of an ambiguous word is represented as a binary vector in which each position indicates the occurrence/absence of a feature, and a single centroid vector is generated for each sense of each word type during training. These centroids are compared with the vectors that represent new examples using the cosine similarity metric. The sense assigned to a given test instance is that of the closest centroid.

As a secondary WSD classifier we use a Support Vector Machine (SVM-Weka), as implemented in the Weka toolkit [19]. SVMs map feature vectors into a high-dimensional space and construct a classifier by searching for the hyperplane in that space that gives the greatest separation between the classes. For our experiments we rely on a polynomial kernel, with the $\mathrm{C}$ parameter set to 1 (the default value in Weka). In both cases, we build a separate classifier for each word type.

Both WSD classifiers rely on an extensive set of features:

- Local collocations (Local): A set of features which describe the context of the ambiguous word token, in 
Table 2 List of target words for our WSD experiment ( $N$ = noun, $\mathrm{V}=$ verb, $\mathrm{J}=$ adjective). We present the number of train and test instances, the number of classes, and the bias of the majority class

\begin{tabular}{|c|c|c|c|c|}
\hline Word & Train \# & Test \# & Classes & \# Top class $\%$ \\
\hline expression. $N$ & 1465 & 265 & 5 & 0.51 \\
\hline transcription.N & 1214 & 210 & 2 & 0.83 \\
\hline activation.N & 1177 & 272 & 3 & 0.89 \\
\hline promoter.N & 770 & 141 & 3 & 0.76 \\
\hline binding.N & 625 & 113 & 2 & 0.84 \\
\hline induce.V & 565 & 128 & 3 & 0.65 \\
\hline activate.V & 523 & 93 & 2 & 0.84 \\
\hline effect.N & 416 & 79 & 4 & 0.83 \\
\hline inhibit.V & 412 & 57 & 2 & 0.67 \\
\hline induction.N & 405 & 116 & 4 & 0.57 \\
\hline bind.V & 373 & 80 & 2 & 0.52 \\
\hline role.N & 342 & 75 & 3 & 0.87 \\
\hline express.V & 308 & 55 & 5 & 0.57 \\
\hline increase.V & 289 & 59 & 2 & 0.57 \\
\hline stimulation.N & 284 & 53 & 4 & 0.89 \\
\hline regulation.N & 274 & 52 & 3 & 0.66 \\
\hline regulate.V & 265 & 49 & 3 & 0.59 \\
\hline require.V & 257 & 53 & 3 & 0.74 \\
\hline production.N & 251 & 45 & 4 & 0.49 \\
\hline inhibition.N & 219 & 38 & 2 & 0.79 \\
\hline mediate.V & 218 & 54 & 3 & 0.74 \\
\hline stimulate.V & 215 & 35 & 3 & 0.83 \\
\hline result.V & 180 & 35 & 4 & 0.86 \\
\hline enhance.V & 178 & 27 & 2 & 0.62 \\
\hline phosphorylation.N & 170 & 52 & 3 & 0.64 \\
\hline increase.N & 157 & 27 & 2 & 0.52 \\
\hline lead.V & 146 & 25 & 2 & 0.83 \\
\hline interaction.N & 144 & 41 & 2 & 0.71 \\
\hline associate.V & 138 & 43 & 2 & 0.85 \\
\hline block.V & 138 & 28 & 3 & 0.67 \\
\hline control.N & 124 & 26 & 2 & 0.87 \\
\hline translocation.N & 123 & 20 & 2 & 0.76 \\
\hline tyrosine.N & 119 & 24 & 2 & 0.76 \\
\hline synthesis.N & 119 & 9 & 3 & 0.75 \\
\hline detect.V & 109 & 16 & 7 & 0.76 \\
\hline tNF.N & 108 & 11 & 2 & 0.70 \\
\hline inducible.J & 108 & 28 & 3 & 0.78 \\
\hline affect.V & 107 & 14 & 4 & 0.62 \\
\hline transactivation.N & 106 & 4 & 2 & 0.86 \\
\hline nucleus.N & 104 & 12 & 2 & 0.85 \\
\hline decrease.V & 98 & 12 & 2 & 0.54 \\
\hline reduce.V & 90 & 26 & 2 & 0.52 \\
\hline control.V & 89 & 17 & 2 & 0.65 \\
\hline suppress.V & 89 & 20 & 2 & 0.65 \\
\hline degradation.N & 88 & 15 & 2 & 0.72 \\
\hline produce.V & 87 & 19 & 3 & 0.55 \\
\hline transcript.N & 80 & 21 & 2 & 0.88 \\
\hline occur.V & 80 & 24 & 2 & 0.89 \\
\hline target.N & 75 & 16 & 3 & 0.87 \\
\hline
\end{tabular}

Table 2 List of target words for our WSD experiment ( $\mathbf{N}$ = noun, $\mathbf{V}=$ verb, $\mathbf{J}=$ adjective). We present the number of train and test instances, the number of classes, and the bias of the majority class (Continued)

\begin{tabular}{lrrrl}
\hline dependent.J & 73 & 16 & 3 & 0.71 \\
cause.V & 72 & 10 & 2 & 0.85 \\
essential.J & 70 & 9 & 2 & 0.84 \\
interact.V & 70 & 15 & 2 & 0.51 \\
heterodimer.N & 69 & 22 & 2 & 0.84 \\
secretion.N & 66 & 11 & 2 & 0.73 \\
prevent.V & 65 & 12 & 2 & 0.60 \\
change.N & 62 & 10 & 3 & 0.81 \\
transfecte.V & 61 & 25 & 3 & 0.85 \\
absence.N & 60 & 10 & 7 & 0.83 \\
modulate.V & 58 & 8 & 3 & 0.79 \\
contribute.V & 55 & 16 & 3 & 0.78 \\
decrease.N & 51 & 10 & 2 & 0.61 \\
cross-linking.N & 50 & 2 & 2 & 0.52 \\
\hline
\end{tabular}

the form of: (1) bigrams and trigrams containing the ambiguous word constructed from lemmas, word forms, POS tags and PROTEIN tags provided in the BioNLP 2009 dataset; and (2) the lemma/word-form of preceding/ following content words (adjectives, adverbs, nouns and verbs) occurring in the same sentence as the target word.

- Syntactic dependencies: We identify the syntactic dependencies between the target word and other words in the sentence. We define two types of features: (1) lexicalised dependencies (the dependency relation type + the related lexical item), and (2) unlexicalised dependencies (the dependency relation type only). We extract the dependencies from the four parsers provided by the BioNLP 2009 shared task organisers [20].

- Bag-of-words (BOW): Lemmas of all content words (nouns, verbs, adjectives, adverbs) in the same sentence as the target word, and, as a separate feature, lemmas of all content words within a \pm 4 -word window around the target word.

- Medical Subject Headings (MeSH) [21]: the manually-assigned $\mathrm{MeSH}$ terms associated with the document that the sentence was taken from. $\mathrm{MeSH}$ is a controlled vocabulary for indexing biomedical and health-related information and documents, and all biomedical papers in MEDLINE are indexed with MeSH data.

We also implement a sequential tagger that does not follow the WSD approach of separate models for each word type. Specifically, we use the CRF++ toolkit [22], which has been shown to be highly successful over various chunking tasks. CRFs provide a discriminative framework for building structured models to segment and label sequence data [23]. CRFs have the well-known advantage that they both model sequential effects and support the use of large numbers of features. 
For the CRF classifier we used a similar set of feature types to the WSD classifiers: word-forms, lemmas, POS, chunk tags, protein annotations, and grammatical dependencies. For dependency annotation, we used the Bikel parser and GDep as provided by the shared task organisers. This information was provided as a feature that expresses the grammatical function of the token. We applied a window size of 4 words in either direction from the target word.

Note that the CRF classifier is able to classify occurrences other than those for the target word types, but we evaluate only over the 63 target words for a fair comparison.

Finally, we also built an extended version of CRF (CRF-VSM) which uses the WSD predictions as an extra feature. In cases where the current token is none of the target words, the feature has the value NULL. For the training data, we obtain the predictions by using 3fold cross-validation, and for the test data we rely on the full training set. This system allows us to combine the NER and WSD approaches to the problem. For evaluation we provide the precision, recall, and F-score for each class, in addition to reporting the micro-averaged results over the 9 trigger-word classes. We also show the average accuracy across all instances; this score is less relevant to the final goal of correctly identifying trigger words, because it is affected by the predominance of the NON-EVENT instances and ignores recall.

We use randomised estimation to calculate whether any performance differences between methods are statistically significant [24]. As a baseline we present the Majority Class (MC) classifier, which assigns the most frequent class seen in the training data to all the test instances.

\section{Feature analysis}

Supervised WSD systems build upon the "one sense per collocation" heuristic, which shows that in fixed collocations, a given word will tend to occur with the same meaning. Yarowsky [15] defined "collocation" as the cooccurrence of words in a given relationship, for instance the words no relevant occurring immediately before the word changes. The features that we used satisfy this definition of "collocation". We analyse the class entropy of the features in our collection and compare it with the WSD corpora introduced in Section. We rely on all features that occur at least twice, and we average the entropy values of all the features for each of the four basic feature types. We did not have access to all feature types for every corpus (e.g. MeSH is not found in Senseval), but the available ones can give us some insight into the differences across the corpora.

The average entropy per feature type and corpus are given in Table 3. Looking at the overall entropy for the
Table 3 Entropy for each feature type across the three WSD corpora

\begin{tabular}{lccc}
\hline Feature type & BioNLP & NLM & Senseval \\
\hline Local & 0.301 & 0.176 & 0.380 \\
Syntactic dep. & 0.305 & - & 0.280 \\
BOW & 0.339 & 0.186 & 0.455 \\
MeSH & 0.360 & 0.183 & - \\
\hline Overall & 0.323 & 0.180 & 0.435 \\
\hline
\end{tabular}

BioNLP corpus, we can see that it falls between the low entropy of the domain-specific NLM and the higher entropy of Senseval. This is encouraging because our classifier has been able to obtain good performance over the Senseval dataset. The scores for the NLM collection show that this dataset is particularly well-suited to WSD, which has been illustrated by the high performance reported in the literature [7].

With respect to the different feature types, there are not big differences in our collection, and as expected, local features and syntactic dependencies do best. More surprising are the results across Senseval data, with the high entropy of BOW and the low score of syntactic dependencies. NLM features have low entropy for all the different types.

We also analysed the entropy for the different target word types in the BioNLP dataset. We grouped the words into three sets based on their average feature entropy, as can be seen in Table 4, together with examples of word types. We expect that words with low entropy will achieve better performance, and we test this idea in Section.

\section{Results}

We analyse the results of the different classifiers over the development portion of the BioNLP dataset. The scores of the four classifiers and the Majority Class (MC) baseline are given in Table 5. SVM-Weka performs poorly, just above the majority class baseline in terms of F-score. We can see that VSM suffers from low precision, and CRF has low recall, but both achieve an F-score quite a bit higher than SVM-Weka, despite SVMs having been found to be the strongest performer by the top two teams in the original shared task. The smaller set of instances used to build

\begin{tabular}{|c|c|c|c|c|}
\hline Average Entropy & \# Words & Avg. Freq. & Example & Major Class \\
\hline$H<.3$ & 28 & 272.8 & occur.V & NON-EVT \\
\hline $.3 \leq H<.4$ & 20 & 167.3 & change.N & NON-EVT \\
\hline$H \geq .4$ & 15 & 264.8 & express.V & GENE-EXP \\
\hline
\end{tabular}


Table 5 WSD performance of the different classifiers (the best results per column are given in bold)

\begin{tabular}{lcccc}
\hline System & Acc & Prec & Rec & F-score \\
\hline MC & 72.8 & 55.9 & 27.4 & 36.7 \\
SVM-Weka & 62.7 & 39.9 & 39.6 & 39.8 \\
CRF & 78.4 & $\mathbf{7 2 . 4}$ & 46.3 & 56.5 \\
VSM & 71.7 & 54.4 & $\mathbf{6 2 . 5}$ & 58.1 \\
CRF-VSM & $\mathbf{7 8 . 9}$ & 70.2 & 52.6 & $\mathbf{6 0 . 1}$ \\
\hline
\end{tabular}

the models could be one of the reasons for the low performance of our SVM. By using only instances from the target word type, the training data for each class is reduced considerably, and the conceptually simpler VSM performs better in this setting. Another reason for the low performance of SVM could be the lack of tuning of the kernel and parameters. The best performer of all, however, in terms of both accuracy and F-score, is the combined CRFVSM, where the output of VSM is incorporated as a feature into CRF. This approach significantly improves the recall and F-score of the base CRF (p-values $<0.01$ ), and it is also significantly better in precision ( $\mathrm{p}$-value $<0.01$ ) and F-score (p-value $<0.05$ ) than the base VSM. That is, the combination of the two approaches performs better than each of the two methods in isolation. This result is highly significant in suggesting a new approach to the BioNLP 2009 task, or at the very least, a new source of features to combine with the existing task 1 systems.

We also compared the performance of CRF and VSM according to the average entropy of the target words. The results are given in Table 6. We can see that VSM obtains the best performance for low-entropy words, and it is able to clearly improve over CRF for words with an average feature entropy below 0.40 . The improvement in recall is statistically significant $(p<$ 0.01 ), and the F-score improvement is statistically significant for words with entropy below $0.30(p<0.05)$. This demonstrates that we have the ability to pre-identify words that are most likely to benefit from WSD. Note also that, surprisingly, the best performance overall is obtained in the high-entropy range.

We also report the results of CRF-VSM by event type in Table 7. We can see that different types exhibit very different performance, and the most difficult to predict

Table 6 WSD result for different entropy ranges (the best score for each evaluation metric and entropy range is shown in bold)

\begin{tabular}{ccccccc}
\hline Average Entropy & \multicolumn{3}{c}{ CRF } & & VSM \\
& Prec & Rec & F-sc. & Prec & Rec & F-sc. \\
\hline$H<.3$ & $\mathbf{7 1 . 6}$ & 25.7 & 37.9 & 39.5 & $\mathbf{5 8 . 3}$ & $\mathbf{4 7 . 1}$ \\
$.3 \leq H<.4$ & $\mathbf{7 6 . 5}$ & 42.1 & 54.3 & 63.3 & $\mathbf{5 6 . 1}$ & $\mathbf{5 9 . 5}$ \\
$H \geq .4$ & $\mathbf{7 0 . 6}$ & 60.9 & $\mathbf{6 5 . 4}$ & 59.9 & $\mathbf{6 9 . 5}$ & 64.3 \\
\hline
\end{tabular}

Table 7 Performance of CRF-VSM by event type, sorted by F-score (Freq. = Frequency of the event in test data)

\begin{tabular}{lrrrr}
\hline Event & Freq. & Prec. & Rec. & F-score \\
\hline PROTEIN CATABOLISM & 13 & 100 & 84.6 & 91.7 \\
GENE EXPRESSION & 208 & 75.9 & 77.4 & 76.7 \\
PHOSPHORYLATION & 34 & 82.8 & 70.6 & 76.2 \\
LOCALIZATION & 13 & 72.7 & 61.5 & 66.7 \\
BINDING & 119 & 78.7 & 52.9 & 63.3 \\
TRANSCRIPTION & 52 & 64.0 & 61.5 & 62.7 \\
POSITIVE REGULATION & 263 & 64.9 & 42.2 & 51.2 \\
REGULATION & 86 & 51.2 & 25.6 & 34.1 \\
NEGATIVE REGULATION & 60 & 50.0 & 23.3 & 31.8 \\
\hline
\end{tabular}

are regulation events. This could happen because these three classes tend to occur in similar contexts, and are hard to differentiate. The best performance is achieved by the rare PROTEIN CATABOLISM event, with full precision and a high F-score of $91.7 \%$; the high-frequency event GENE EXPRESSION ranks second with an F-score of $76.7 \%$.

Finally, we present the overall results for each POS in Table 8 . The main observation is that the F-score and recall are much higher for nouns than for verbs, but the precision and accuracy for the two are similar.

The difficulty of disambiguating verb senses has been observed variously in the WSD literature, and these results seem to confirm that tendency. Regarding adjectives, the performance is actually below the majority class baseline, but there are only three word types and relatively few token instances for each, so the overall impact on results is negligible. The difficulty here appears to relate to the choice of which word to annotate as the trigger word, and a richer model of the interaction of the words related to the event would be required to improve the performance.

\section{Discussion}

Our results over the BioNLP 2009 dataset show that the transformation of existing biomedical annotation into a WSD dataset can be challenging. One of the main differences over standard WSD is that the word-models tend to be biased towards the non-event class, and this can be problematic for the classifiers. For the noun transcription, e.g., $83 \%$ of the training instances are of type NON-EVENT. A possible solution could be to

Table 8 WSD experiment performance of VSM by POS. The best result per column is given in bold

\begin{tabular}{lrrrrr}
\hline POS & \# Test & Acc & Prec & Rec & F-score \\
\hline Noun & 1802 & $\mathbf{7 2 . 9}$ & $\mathbf{5 5 . 8}$ & $\mathbf{7 0 . 0}$ & $\mathbf{6 2 . 1}$ \\
Verb & 1055 & 70.0 & 53.2 & 53.2 & 53.2 \\
Adj. & 53 & 64.2 & 9.1 & 10.0 & 9.5 \\
\hline
\end{tabular}


apply re-sampling techniques to build more balanced models [25].

Another issue is the difficulty of event annotation for humans, even when strategies to ensure quality are put in place. After a long process, the BioNLP event annotation enforced the following guidelines [3]: text-bound annotation (grounding all annotations to strings in text), single-facet annotation (keeping the viewpoint of annotation simple and very focused), and semantic typing (looking at types of entities for each event, and types of event for each entity to detect anomalies). The developers of the corpus explain that this process improved the inter-annotator agreement significantly, although they did not provide the numbers. They also describe how some high-frequency words can represent a wide variety of biomedical events depending on their related words in the context.

In our error analysis we found that meta-linguistic knowledge would be necessary to correctly identify some of the targeted events, and richer features than standard WSD sets would be required. For instance, let us consider the noun transcription and the event type TRANSCRIPTION, which refers to the process of creating an equivalent RNA copy of a sequence of DNA. The word occurs 201 times as the TRANSCRIPTION event, and 1,013 times as NON-EVENT in the training data. Looking at the features, they have an average entropy of 0.23 , which puts this target word among the lowentropy words. However, for this word the F-score of VSM is slightly lower than CRF, and we can see that some of the NON-EVENT occurrences have similar features to the event occurrences. By analysing the examples, we found the following phenomena, which are not captured by our current model:

1. The event is described in the text as a process, and the annotators mark the word that culminates it, not the initiator. In this example, lack is annotated instead of transcription:

The transcription was initiated from one of three EBNA promoters, Qp: by contrast, both $\mathrm{Cp}$ and Wp were silent, thus resulting in the [TRANslack] of EBNA2 mRNA.

2. The event is underspecified in the sentence, referring to an unspecified gene, and it is not considered relevant. In this example, transcription is not annotated:

OTF-1-enriched protein fractions did not affect DRA gene transcription although it functionally enhanced the transcription of another gene.

3. Multiple events occur simultaneously, and the annotation has to accommodate their relationship. In the following example the main event is a negative regulation event (NEG-REG) that affects a transcription event. The annotators seem to focus on the surface form of the
NEG-REG event first (marking destabilization), and then annotate as TRANSCRIPTION the noun phrase that is directly related to it, choosing preformed, and ignoring the first mention of transcription:

Glucocorticoids are known to downregulate interleukin-1 beta production in monocytic cells by two different mechanims: direct inhibition of the gene transcription and [NEG-REG destabilization] of the [TRANSpreformed] interleukin-1 beta mRNA.

4. The noun transcription occurs multiple times referring to the same event, but only the first occurrence is tagged:

A 2-4-fold increase in IFN-beta promoter [TRANStranscription] was observed in Sendai virus induced extracts, and deletion of PRDI and PRDII elements decreased this induced level of transcription.

These examples illustrate that for the BioNLP 2009 dataset, there are meta-linguistic aspects of the annotation that have to be taken into account, and more consistency is required to close the gap between textual representations and the ultimate goal of biomedical pathways. Significant effort has been done in the annotation of the BioNLP dataset, but we believe that wordby-word analysis can provide better means to improve and extend this kind of tagging. It has been shown in the WSD evaluation tracks that the annotation of lexical-sample datasets is easier and produces better quality data than all-words datasets, and this could be translated to the annotation of biomedical events. We have seen in this corpus that 63 ambiguous word types cover $62 \%$ of the event annotations, and focusing on the instances of these words separately could be a better way to produce consistent annotation.

\section{Conclusions}

We described a WSD-based method for detecting event trigger words in the BioNLP 2009 shared task data, and demonstrated that it attains superior performance than a traditional sequential tagging approach. The highest score is achieved when using the WSD predictions as features for a sequential tagger, which significantly improves the recall and F-score of the latter. We also observed that measuring the training class-entropy of features seems to be a good indicator of the kind of target word types that can improve over a sequential tagger.

Another result of this work is the identification of consistency issues in biomedical annotation, even when clear guidelines are provided. We found that a wordcentered approach may help to find inconsistencies, specially given that a few target words seem to have high coverage of the trigger annotations. For future work we are planning to explore other challenges, such as BioCreative, and also to deploy full systems for the BioNLP 
shared task challenge, in order to directly compare against other systems.

\section{Acknowledgements}

NICTA is funded by the Australian Government as represented by the Department of Broadband, Communications and the Digital Economy and the Australian Research Council through the ICT Centre of Excellence program.

This article has been published as part of BMC Bioinformatics Volume 12 Supplement 2, 2011: Fourth International Workshop on Data and Text Mining in Bioinformatics (DTMBio) 2010. The full contents of the supplement are available online at http://www.biomedcentral.com/1471-2105/12? issue $=\mathrm{S} 2$.

\section{Author details}

'NICTA VRL, The University of Melbourne, VIC 3010, Australia. ${ }^{2}$ Department of Computer Science and Software Engineering, The University of Melbourne, VIC 3010, Australia.

\section{Competing interests}

The authors declare that they have no competing interests.

Published: 29 March 2011

\section{References}

1. Text REtrieval Conference (TREC). [http://trec.nist.gov].

2. John Wilbur, Lawrence Smith, Lorraine Tanabe: Biocreative 2. gene mention task. Second BioCreative Evaluation Workshop Madrid, Spain; 2007, 7-16.

3. Jin-Dong Kim, Tomoko Ohta, Jun'Ichi Tsujii: Corpus annotation for mining biomedical events from literature. BMC Bioinformatics 2008, 9:10.

4. Jin-Dong Kim, Tomoko Ohta, Sampo Pyysalo, Yoshinobu Kano, Jun'ichi Tsujii: Overview of BioNLP'09 shared task on event extraction. Proceedings of the BioNLP 2009 Workshop Companion Volume for Shared Task Boulder, USA; 2009, 1-9.

5. Proceedings of the Fourth International Workshop on Semantic Evaluations (SemEval-2007). In Association for Computational Linguistics. Prague, Czech Republic;Eneko Agirre, Lluis Màrquez, Richard Wicentowski 2007.

6. Lluis Màrquez, Gerard Escudero, David Martinez, German Rigau: Supervised corpus-based methods for word sense disambiguation. In Word Sense Disambiquation. Springer, Dordrecht, Netherlands; Eneko Agirre and Phil Edmonds 2006.

7. Mark Stevenson, Yikun Guo, Robert Gaizauskas, David Martinez: Disambiguation of biomedical text using diverse sources of information. BMC Bioinformatics 2008, 9(Suppl 11):S7.

8. Eneko Agirre, Oier Lopez de Lacalle, Christiane Fellbaum, Andrea Marchetti, Antonio Toral, Piek Vossen: Semeval-2010 task 17: all-words word sense disambiguation on a specific domain. DEW '09: Proceedings of the Workshop on Semantic Evaluations: Recent Achievements and Future Directions 2009, 123-128.

9. Marc Weeber, James G Mork, Alan R Aronson: Developing a test collection for biomedical word sense disambiguation. Proceedings of the 2001 AMIA Symposium Washington DC, USA; 2001, 746-750.

10. BioCreAtlvE challenge. [http://biocreative.sourceforge.net/index.html].

11. Jari Björne, Juho Heimonen, Filip Ginter, Antti Airola, Tapio Pahikkala, Tapio Salakoski: Extracting complex biological events with rich graphbased feature sets. Proceedings of the BioNLP 2009 Workshop Companion Volume for Shared Task Boulder, USA; 2009, 10-18.

12. Ekaterina Buyko, Erik Faessler, Joachim Wermter, Udo Hahn: Event extraction from trimmed dependency graphs. Proceedings of the BioNLP 2009 Workshop Companion Volume for Shared Task Boulder, USA; 2009, 19-27.

13. Diana McCarthy: Word sense disambiguation: An overview. Language and Linguistics Compass 2009, 3(2):537-558.

14. Roberto Navigli: Word sense disambiguation: a survey. ACM Computing Surveys 2009, 41(2):1-69.

15. David Yarowsky: One sense per collocation. HLT '93: Proceedings of the workshop on Human Language Technology Princeton, USA; 1993, 266-271.
16. Rada Mihalcea, Timothy Chklovski, Adam Kilgarriff: The Senseval-3 English lexical sample task. Senseval-3: Third International Workshop on the Evaluation of Systems for the Semantic Analysis of Text Barcelona, Spain; 2004, 25-28.

17. Eneko Agirre, David Martinez: The Basque Country University system: English and Basque tasks. Proceedings of the $3 r d$ ACL workshop on the Evaluation of Systems for the Semantic Analysis of Text (SENSEVAL) Barcelona, Spain; 2004, 44-48.

18. Yoshimasa Tsuruoka, Yuka Tateishi, Jin-Dong Kim, Tomoko Ohta, John McNaught, Sophia Ananiadou, Jun'ichi Tsujii: Developing a robust part-of-speech tagger for biomedical text. Advances in Informatics - 10th Panhellenic Conference on Informatics Volas, Greece; 2005, 382-392.

19. Ian H Witten, Eibe Frank: Data Mining: Practical machine learning tools and techniques. Morgan Kaufmann, San Francisco, USA; 2005.

20. BioNLP 2009 shared task. [http://www-tsujii.is.s.u-tokyo.ac.jp/GENIA/ SharedTask/tools.shtml].

21. Stuart J Nelson, Tammy Powell, Betsy L Humphreys: The Unified Medical Language System (UMLS) project. In Encyclopedia of Library and Information Science. third edition. CRC Press, Boca Raton, USA;Marcia J. Bates and Mary Niles Maack 2002.

22. CRF++: Yet Another CRF toolkit. [http://crfpp.sourceforge.net/].

23. Charles Sutton, Andrew Mccallum: Introduction to conditional random fields for relational learning. In Introduction to Statistical Relational Learning. MIT Press, Cambridge, USA;Lise Getoor and Ben Taskar 2006.

24. Alexander Yeh: More accurate tests for the statistical significance of result differences. Proceedings of the 18th Conference on Computational Linquistics (COLING) Saarbrücken, Germany; 2000, 947-953.

25. Sotiris Kotsiantis, Dimitris Kanellopoulos, Panayiotis Pintelas: Handling imbalanced datasets: A review. GESTS International Transactions on Computer Science and Engineering 2006, 30:25-36.

doi:10.1186/1471-2105-12-S2-S4

Cite this article as: Martinez and Baldwin: Word sense disambiguation for event trigger word detection in biomedicine. BMC Bioinformatics 2011 12(Suppl 2):S4.

\section{Submit your next manuscript to BioMed Central and take full advantage of:}

- Convenient online submission

- Thorough peer review

- No space constraints or color figure charges

- Immediate publication on acceptance

- Inclusion in PubMed, CAS, Scopus and Google Scholar

- Research which is freely available for redistribution

Submit your manuscript at www.biomedcentral.com/submit
Ciomed Central 